\title{
Reduction of the common mode voltage of a matrix converter fed direct torque control
}

\author{
Carlos Ortega $^{1 \mathrm{a})}$, Antoni Arias ${ }^{2}$, Cedric Caruana ${ }^{3}$, \\ and Maurice Apap $^{3}$ \\ ${ }^{1}$ Escola Universitria Salesiana de Sarrià \\ Pg. Sant Joan Bosco, 74, Barcelona 08017, Spain \\ ${ }^{2}$ Universitat Politcnica de Catalunya \\ Colom, 1, Terrassa 08222, Spain \\ ${ }^{3}$ University of Malta \\ MSD, 2080, Malta \\ a) cortega@euss.es
}

\begin{abstract}
The work presented in this paper proposes an approach to reduce the common mode voltage at the output of matrix converters when a direct torque control scheme is employed to drive a permanent magnet synchronous motor. A mathematical analysis of the common mode voltage at the output of matrix converters is firstly carried out which is then corroborated by simulation results. Based on this analysis, a very simple algorithm to reduce the common mode voltage is proposed and investigated. Finally, experimental results corroborating the effectiveness of the proposed method are shown.
\end{abstract}

Keywords: matrix converters, common mode voltage, direct torque control

Classification: Electromagnetic compatibility (EMC)

\section{References}

[1] D. A. Rendusara and P. N. Enjet, "An improved inverter output filter configuration reduces common and differential modes $\mathrm{dv} / \mathrm{dt}$ at the motor terminals in pwm drive systems," IEEE Trans. Power Electron., vol. 13, pp. 1135-1143, Nov. 1998.

[2] A. L. Julian, G. Oriti, and T. A. Lipo, "Elimination of common-mode voltage in three-phase sinusoidal power converters," IEEE Trans. Power Electron., vol. 14, pp. 982-989, Sept. 1999.

[3] M. Venturini and A. Alsina, "Analysis and design of optimum-amplitude nine-switch direct ac-ac converters," IEEE Trans. Power Electron., vol. 4, pp. 101-112, Jan. 1989.

[4] H. J. Cha and P. N. Enjeti, "An Approach to Reduce Common-Mode Voltage in Matrix Converter," IEEE Trans. Ind. Appl., vol. 39, pp. 11511159, July/Aug. 2003.

[5] D. Casadei, G. Serra, and A. Tani, "The Use of Matrix Converters in Direct Torque Control of Induction Machines," IEEE Trans. Ind. Electron., 
vol. 48, pp. 1057-1064, Dec. 2001.

[6] L. Empringham, P. Wheeler, and J. L. Clare, "Intelligent Commutation of Matrix Converter Bi-directional Switch Cells using Novel Gate Drive Techniques," Proc. PESC '98, vol. 1, pp. 707-713, June/July 1998.

\section{Introduction}

The Common Mode Voltage (CMV) produced by modern power converters is considered as one of the main sources of early motor winding failure and bearing deterioration. High frequency components and large amplitudes of the CMV at the motor neutral point have been shown to generate high frequency currents to the ground path and induced shaft voltage [1]. Strategies to reduce the CMV at the output of standard Voltage Source Inverter (VSI) have been proposed in the literature [2]. In the recent past, Matrix Converters (MCs) [3] have received considerable attention as they are becoming a good alternative to the VSI. In [4], a new modulation strategy of the MC which reduces the CMV is presented. Based on the Indirect Space Vector Modulation (ISVM) technique, the method selects a medium value phase voltage as a zero vector and places it in the center or on the both sides of the sampling period without changing the active voltage vectors. In [5], the use of a MC to implement a Direct Torque Control (DTC) of an Induction Motor (IM) was introduced.

In the last decade, DTC has emerged to become an attractive alternative to the well-known Field Oriented Control (FOC). A very good performance, obtaining results as good as the classical FOC but with several advantages based on its simpler structure and control diagram, is one of its main characteristics.

This paper analyzes the CMV at the output of MCs. The mathematical analysis is confirmed by simulation results. Based on this analysis and the approach presented in [4], an algorithm to reduce the CMV when DTC is employed to drive a Permanent Magnet Synchronous Motor (PMSM) is developed. The algorithm is based on the selection of proper zero vectors without altering the original DTC using MC described in [5]. The effectiveness of the method is validated by experimental results which, in contrast to those obtained in [4], show that the CMV do not depend on the output frequency.

\section{Common Mode Voltage in Matrix Converters}

A MC connected to a PMSM is shown in Fig. 1 . The impedance $Z_{c m}$ represents the leakage current path between the machine's neutral point $(n)$ and ground $(N)$.

The CMV is defined as the voltage between these two points:

$$
v_{c m}=v_{n N}
$$




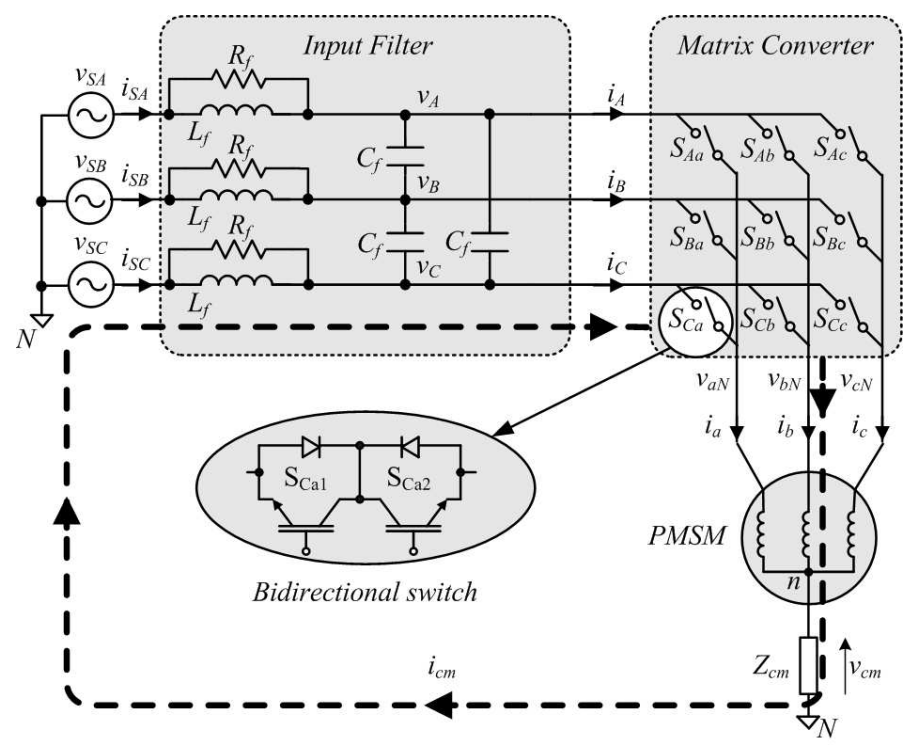

Fig. 1. $3 \times 3$ Matrix Converter.

Taking into consideration the CMV $v_{c m}$, the voltage expressions of the PMSM can be written as:

$$
\begin{aligned}
& v_{a N}-v_{c m}=R_{s} i_{a}+L_{s} \frac{d i_{a}}{d t}+\frac{d}{d t} \psi_{a} \\
& v_{b N}-v_{c m}=R_{s} i_{b}+L_{s} \frac{d i_{b}}{d t}+\frac{d}{d t} \psi_{b} \\
& v_{c N}-v_{c m}=R_{s} i_{c}+L_{s} \frac{d i_{c}}{d t}+\frac{d}{d t} \psi_{c}
\end{aligned}
$$

where $v_{a N}, v_{b N}$, and $v_{c N}$ are the MC output voltages with respect to ground $(N) . R_{s}$ and $L_{s}$ are the resistance and inductance of each phase of the PMSM respectively. Assuming a balanced induced voltage system $\psi_{a}+\psi_{b}+\psi_{c} \approx 0$ and the fact that $i_{a}+i_{b}+i_{c} \approx 0$, the CMV at the output of the MC can be found by adding (2), (3) and (4):

$$
v_{c m}=\frac{v_{a N}+v_{b N}+v_{c N}}{3}
$$

When active vectors are selected in a MC, there are two output phases connected to the same input phase [6]. Thus, the CMV when active vectors are delivered by a $\mathrm{MC}$ can be written as:

$$
v_{c m}^{a}=\frac{v_{i}+2 v_{j}}{3}
$$

where $i$ and $j$ represent the two input phases involved when an active vector (superscript $a$ ) is delivered by the MC.

When the input filter is properly designed, the phase voltages at the input side of the MC $v_{A}, v_{B}$, and $v_{C}$ can be considered equal to the phase voltages of the grid $v_{S A}, v_{S B}$, and $v_{S C}$ respectively [6]. Assuming a balanced voltage at the grid and, hence, at the input side of the MC,

$$
v_{S A}=v_{A}=V_{p} \sin (\omega t)
$$




$$
\begin{aligned}
& v_{S B}=v_{B}=V_{p} \sin \left(\omega t-\frac{2 \pi}{3}\right) \\
& v_{S C}=v_{C}=V_{p} \sin \left(\omega t+\frac{2 \pi}{3}\right)
\end{aligned}
$$

where $V_{p}$ is the voltage peak value per phase, (6) can be written as:

$$
v_{c m}^{a}=\frac{1}{3} V_{p} \sin (\omega t)+\frac{2}{3} V_{p} \sin \left(\omega t \pm \frac{2 \pi}{3}\right)
$$

Rearranging (10), a simpler form of $v_{c m}^{a}$ is obtained:

$$
v_{c m}^{a}=\frac{1}{\sqrt{3}} V_{p} \sin \left(\omega t \pm \frac{\pi}{2}\right)
$$

Equation (11) states that the instantaneous value of the CMV $v_{c m}^{a}$, when an active vector is delivered by the MC depends on the instantaneous value of the input voltages selected to deliver such vector. The maximum instantaneous value of the CMV $v_{c m}^{a}$, when active vectors are delivered by the MC will be:

$$
v_{c m_{\max }}^{a}= \pm \frac{1}{\sqrt{3}} V_{p}
$$

The CMV will reach its maximum value $v_{c m_{\max }}^{a}$ every $\pm k \pi$ radians.

Each active vector contributes to the CMV as shown in Fig. 2 (a) and (b), where $v_{S A}, v_{S B}$, and $v_{S C}$ are the grid voltages with a frequency of $50 \mathrm{~Hz}$. During each input voltage period $(T=0.02 \mathrm{~s})$, a different output vector is delivered by the MC as shown in Fig. 2 (a) and Fig. 2 (b), where $( \pm 1 \ldots \pm 9)$ are the MC active vectors as defined in [5]. As it can be seen, for every active vector, the CMV is at its maximum value, $\pm \frac{1}{\sqrt{3}} V_{p}$, in every $\pm k \pi$ radians.

When a zero vector is selected in a MC, the three output phases are connected to the same input phase. Thus, when a zero vector is delivered by a MC, the CMV $v_{c m}^{z}$ will be equal to the selected input voltage:

$$
v_{c m}^{z}=v_{i}
$$

where $i$ denotes the selected input phase.
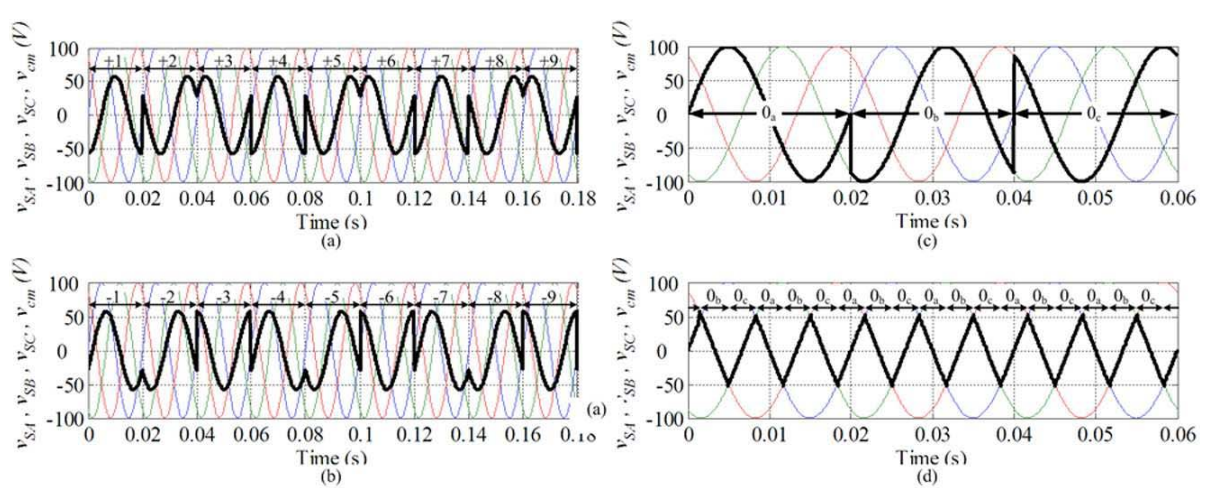

Fig. 2. Common mode voltage. (a) and (b) when active vectors are delivered by a MC. (c) when zero vector are delivered by the MC. (d) Selecting the phase voltage with minimum absolute value. 
Since $v_{i}=V_{p} \sin (w t \pm \phi)$, the maximum CMV $v_{c m}^{z}$ when a zero is selected will be:

$$
v_{c m_{\max }}^{z}= \pm V_{p}
$$

The contribution to the CMV of each zero vector is shown in Fig. 2 (c), where a different zero vector is selected in every input voltage period $(T=$ $0.02 \mathrm{~s})$. It can be noted how the CMV is equal to the amplitude of the selected input phase to deliver the zero vector, being the maximum value of the CMV equal to the peak value of that phase.

\section{Reduction of the Common mode Voltage}

In DTC schemes, a zero vector is usually selected when the actual electromagnetic torque is within the corresponding comparator hysteresis bands [5]. In a MC, a zero vector is delivered by connecting all the output phases to the same input phase. Since there are three input phases supplying the MC, three different zero vectors can be delivered depending on which input phase is connected to the three output phases. This degree of freedom can be exploited to reduce the CMV by proper selection of the zero vectors.

When an input phase voltage reaches its positive or negative peak value $\pm V_{p}$, the other two phases will be at $\pm \frac{1}{2} V_{p}$. Hence, the maximum CMV can be reduced to $\pm \frac{1}{2} V_{p}$ if the input phase voltage with minimum absolute value is selected to deliver the required zero vector. The contribution to the CMV of zero vectors when the input phase with minimum absolute value is selected to deliver a zero vector is shown in Fig. 2 (d). When a zero vector is required by the DTC algorithm, the system computes the absolute value of every input phase and selects the one with the minimum value. The selected phase will be the one connected to the three output phases to deliver the zero vector. In contrast with the ISVM described in [4], where the selection of zero vectors with the minimum absolute value can not always be ensured due to the varying nature of the input voltage and the longer time during which zero vectors are applied at low output frequency, the proposed method ensures the selection of the most suitable zero vector irrespective of the output frequency.

\section{Experimental Results}

In order to validate the theoretical analysis, experimental tests have been carried out employing a PMSM with the following parameters: number of pole pairs $p=4$, power $P=200 \mathrm{~W}$, torque $T_{e}=0.64 \mathrm{Nm}$, speed $N=3000 \mathrm{rpm}$, permanent magnet flux $\psi_{P M}=0.046 \mathrm{~Wb}$, quadrature axis impedance $L_{q}=$ $8.6 \mathrm{mH}$. The proposed method to reduce the CMV is analyzed and compared with the classical DTC using MC proposed in [5], in which the reduction of the number of switching is used as the criteria to select zero vectors. The DTC settings in both experiments are: $T_{e}^{*}=0.64 \mathrm{Nm}$ (rated torque), $\left|\psi^{s *}\right|=\sqrt{\left[\psi_{P M}^{2}+\left(\frac{2}{3} \frac{T_{e}^{*} L_{q}^{s}}{p \psi_{P M}}\right)^{2}\right]}, B T_{e}= \pm 0.015 \mathrm{Nm}, B \psi^{s}= \pm 0.0006 \mathrm{~Wb}$, where $T_{e}^{*}$ is the torque reference, $\left|\psi^{s *}\right|$ is the flux reference, and $B T_{e}$ and $B \psi^{s}$ are the torque and flux hysteresis bands respectively. 
The CMV when the classical DTC using MC is used to drive the PMSM is shown in Fig. 3 (a). The maximum CMV value $v_{c m_{\max }}$ reaches $\pm 100 \mathrm{~V}$, corresponding to the input phase negative and positive peak values. As it has been explained in section 2 , this occurs when an input phase crossing its peak value is selected to deliver a zero vector.

When the proposed method is used under the same conditions, the maximum CMV value is reduced to $\pm 57.7 \mathrm{~V}$, as shown in Fig. 3 (b), corresponding to $\pm 1 / \sqrt{3}$ of the input phase peak value when active vectors are selected.
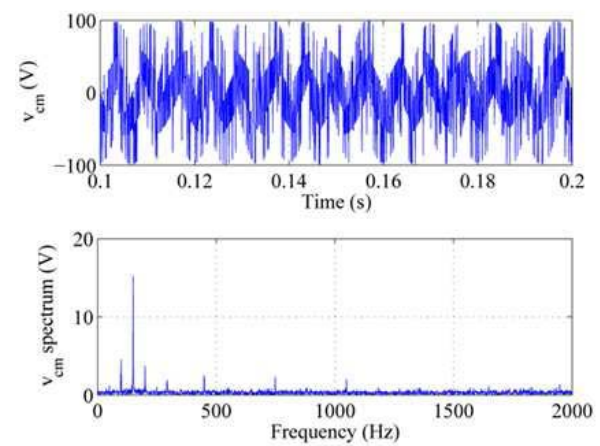

(a)

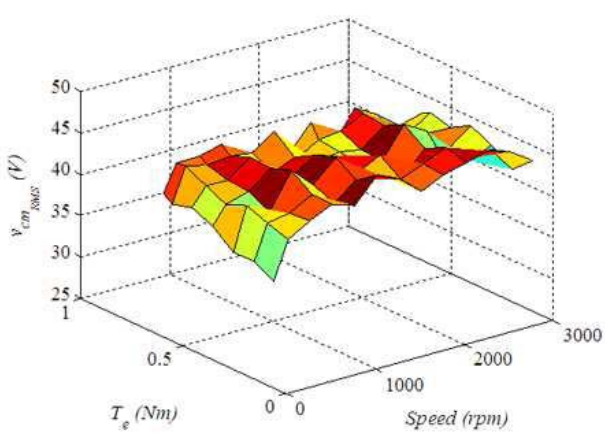

(c)
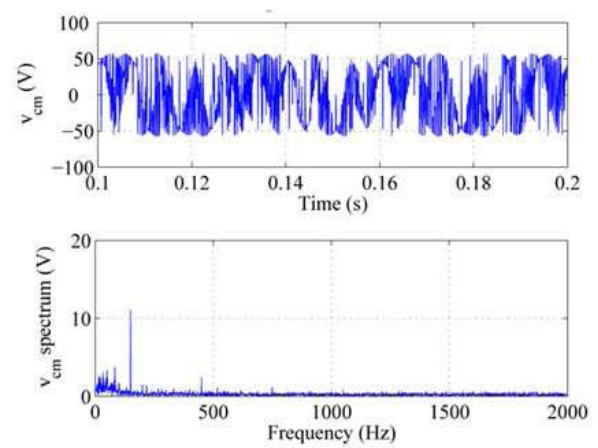

(b)

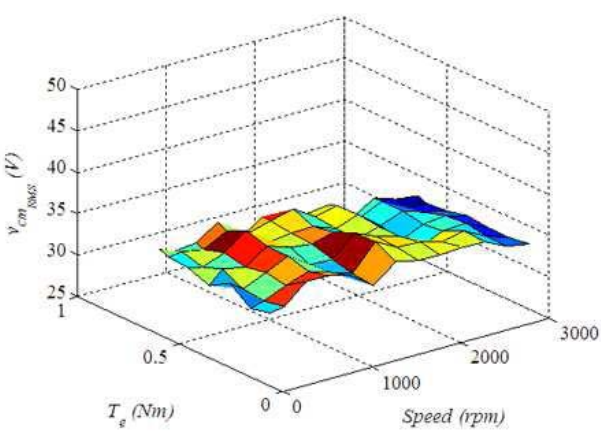

(d)

Fig. 3. Comparison between the classical and the proposed methods. (a) CMV and its spectrum using the classical method. (b) CMV and its spectrum using the proposed method. (c) $v_{c m_{R M S}}$ using the classical method. (d) $v_{c m_{R M S}}$ using the proposed method.

In addition to a reduction of the motor winding failure and degradation of its bearings due to the reduction in the CMV [2], comparing the harmonic spectrum of $v_{c m}$ shown in Fig. 3 (a) and Fig. 3(b), it can be said that the better performance shown by the proposed method will reduce the motor shaft induced voltage [1].

Several experimental tests, covering all the machine operating points, have been carried out in order to deeply compare both methods. The speed has been varied from $100 \mathrm{rpm}$ up to $3000 \mathrm{rpm}$ (rated speed), whereas the torque reference has been varied from $0.1 \mathrm{Nm}$ up to $0.64 \mathrm{Nm}$ (rated torque). In order to assess the performance of both systems, only the RMS value of the CMV $v_{c m_{R M S}}$ has been considered since its peak value is expected to 
remain constant irrespective of the operating conditions.

The results, comparing the classical and the proposed method, are shown in Fig. $3(\mathrm{c})$ and Fig. $3(\mathrm{~d})$ respectively. It is noted that the better performance of the proposed method were verified at over all the operating points. Moreover, in contrast with the results obtained in [4], the flatness of both figures denotes that the RMS value of the CMV $v_{c m_{R M S}}$ does not depend neither on torque nor on speed.

\section{Conclusion}

The common mode voltage produced by MCs has been analyzed focusing on the DTC driving PMSMs. The contribution of zero vectors to the CMV has been shown to be greater than that of active vectors. The advantages of the MCs, such as the availability to deliver three different zero vectors, have been exploited to reduce the CMV in DTC drives. A very simple and effective zero vector selector has been developed allowing a reduction of the maximum CMV in $42.3 \%$ while not deteriorating the output harmonic content. Moreover, the better harmonic spectrum of the CMV, specially at high frequencies, indicates that there will be a lower level of common mode current contributing to extend the machine's operational life. 